\title{
Severity of Disease Among Adults Hospitalized with Laboratory-Confirmed COVID-19 Before and During the Period of SARS-CoV-2 B.1.617.2 (Delta) Predominance - COVID-NET, 14 States, January-August 2021
}

\author{
Christopher A. Taylor, $\mathrm{PhD}^{1}$; Kadam Patel, $\mathrm{MPH}^{1,2}$; Huong Pham, $\mathrm{MPH}^{1}$; Michael Whitaker, $\mathrm{MPH}^{1}$; Onika Anglin, $\mathrm{MPH}^{1,2}$; \\ Anita K. Kambhampati, MPH ${ }^{1}$; Jennifer Milucky, MSPH ${ }^{1}$; Shua J. Chai, MD ${ }^{3,4}$; Pam Daily Kirley, MPH ${ }^{4}$; Nisha B. Alden, MPH ${ }^{5}$; \\ Isaac Armistead, MD5; James Meek, MPH ${ }^{6}$; Kimberly Yousey-Hindes, $\mathrm{MPH}^{6}$; Evan J. Anderson, MD,8,9; Kyle P. Openo, DrPH',8; \\ Kenzie Teno, MPH ${ }^{10}$; Andy Weigel ${ }^{10}$; Maya L. Monroe, MPH ${ }^{11}$; Patricia A. Ryan, MS ${ }^{11}$; Justin Henderson, MPH ${ }^{12}$; \\ Val Tellez Nunez, $\mathrm{MPH}^{12}$; Erica Bye, MPH${ }^{13}$; Ruth Lynfield, MD ${ }^{13}$; Mayvilynne Poblete, MA, MPH ${ }^{14}$; Chad Smelser, MD ${ }^{15}$; \\ Grant R. Barney, $\mathrm{MPH}^{16}$; Nancy L. Spina, MPH ${ }^{16}$; Nancy M. Bennett, MD ${ }^{17}$; Kevin Popham, MPH $^{18}$; Laurie M. Billing, MPH ${ }^{19}$; \\ Eli Shiltz, MPH ${ }^{19}$; Nasreen Abdullah, MD 20 ; Melissa Sutton, MD ${ }^{20}$; William Schaffner, MD ${ }^{21}$; H. Keipp Talbot, MD ${ }^{21}$; Jake Ortega, MPH ${ }^{22}$; \\ Andrea Price ${ }^{22}$; Shikha Garg, $\mathrm{MD}^{1}$; Fiona P. Havers, $\mathrm{MD}^{1}$; COVID-NET Surveillance Team
}

On October 22, 2021, this report was posted as an MMWR Early Release on the MMWR website (https://www.cdc.gov/mmwr). In mid-June 2021, B.1.671.2 (Delta) became the predominant variant of SARS-CoV-2, the virus that causes COVID-19, circulating in the United States. As of July 2021, the Delta variant was responsible for nearly all new SARS-CoV-2 infections in the United States. ${ }^{*}$ The Delta variant is more transmissible than previously circulating SARS-CoV-2 variants (1); however, whether it causes more severe disease in adults has been uncertain. Data from the CDC COVID-19-Associated Hospitalization Surveillance Network (COVID-NET), a population-based surveillance system for COVID-19-associated hospitalizations, were used to examine trends in severe outcomes in adults aged $\geq 18$ years hospitalized with laboratory-confirmed COVID-19 during periods before (January-June 2021) and during (July-August 2021) Delta variant predominance. COVID-19-associated hospitalization rates among all adults declined during January-June 2021 (pre-Delta period), before increasing during July-August 2021 (Delta period). Among sampled nonpregnant hospitalized COVID-19 patients with completed medical record abstraction and a discharge disposition during the pre-Delta period, the proportion of patients who were admitted to an intensive care unit (ICU), received invasive mechanical ventilation (IMV), or died while hospitalized did not significantly change from the pre-Delta period to the Delta period. The proportion of hospitalized COVID-19 patients who were aged 18-49 years significantly increased, from $24.7 \%$ (95\% confidence interval $[\mathrm{CI}]=23.2 \%-26.3 \%$ ) of all hospitalizations in the pre-Delta period, to $35.8 \%$ $(95 \% \mathrm{CI}=32.1 \%-39.5 \%, \mathrm{p}<0.01)$ during the Delta period. When examined by vaccination status, $71.8 \%$ of COVID-19-associated hospitalizations in the Delta period were in unvaccinated adults. Adults aged 18-49 years accounted for $43.6 \%(95 \% \mathrm{CI}=39.1 \%-48.2 \%)$ of all hospitalizations among unvaccinated adults during the Delta period.

\footnotetext{
*https://covid.cdc.gov/covid-data-tracker/\#variant-proportions
}

No difference was observed in ICU admission, receipt of IMV, or in-hospital death among nonpregnant hospitalized adults between the pre-Delta and Delta periods. However, the proportion of unvaccinated adults aged 18-49 years hospitalized with COVID-19 has increased as the Delta variant has become more predominant. Lower vaccination coverage in this age group likely contributed to the increase in hospitalized patients during the Delta period. COVID-19 vaccination is critical for all eligible adults, including those aged $<50$ years who have relatively low vaccination rates compared with older adults.

COVID-NET conducts population-based surveillance for laboratory-confirmed COVID-19-associated hospitalizations in 99 counties across 14 states. ${ }^{\dagger}$ Among residents of a predefined surveillance catchment area, COVID-19-associated hospitalizations are defined as a positive real-time reverse transcription-polymerase chain reaction or rapid antigen detection test result for SARS-CoV-2 during hospitalization or within the 14 days preceding admission. ${ }^{\S}$ Unadjusted age-specific monthly population-based hospitalization rates (hospitalizations per 100,000 persons) among all adults aged $\geq 18$ years irrespective of pregnancy status during JanuaryAugust 2021 were calculated by dividing the total number of hospitalized COVID-19 patients by population estimates within each age group in the surveillance catchment area.9 Using previously described methods (2), clinical outcomes data were collected on a representative sample of hospitalized adults stratified by age and site of admission during

\footnotetext{
$\dagger$ Selected counties in California, Colorado, Connecticut, Georgia, Iowa, Maryland, Michigan, Minnesota, New Mexico, New York, Ohio, Oregon, Tennessee, and Utah can be found at https://www.cdc.gov/mmwr/volumes/69/ $\mathrm{wr} / \mathrm{mm} 6915 \mathrm{e} 3 . \mathrm{htm}$.

$\$$ methods.html

Sates cannot be stratified by pregnancy status because the underlying population of pregnant women in the catchment area is unknown. Rates are calculated using the National Center for Health Statistics' vintage 2019 bridged-race postcensal population estimates for the counties included in surveillance. https:// www.cdc.gov/nchs/nvss/bridged_race.htm
} 
January-August 2021. Using a standardized case report form, trained surveillance staff members abstracted data on sampled cases (updated monthly) from medical charts that included a discharge disposition. Pregnant women (496) were excluded from the analysis because reasons for hospital admission (3) and standards for ICU admission might differ from those for nonpregnant persons. Severe outcomes assessed included ICU admission, receipt of IMV, and all cause in-hospital death. Severe outcomes were compared during periods before (preDelta period) and during Delta variant predominance (Delta period). Because COVID-19 vaccination might affect clinical outcomes (4), and vaccination coverage changed during the study period, results were analyzed overall and stratified by COVID-19 vaccination status. ${ }^{* *}$ Vaccination status was determined using state immunization information systems data $(5,6)$. Variances were estimated using Taylor series linearization method. Chi-square testing was used to compare differences between the pre-Delta and Delta periods; p-values $<0.05$ were considered statistically significant, adjusted for multiple comparisons using the Bonferroni correction method. Unless otherwise noted, percentages presented are weighted to account for the probability of selection for sampled cases (2). All analyses were conducted using SAS statistical software survey procedures (version 9.4; SAS Institute). This activity was reviewed by CDC and was conducted consistent with applicable federal law and CDC policy. ${ }^{\dagger \dagger}$

Based on 87,879 COVID-19 hospitalizations among all adults during January 1-August 31, 2021, irrespective of pregnancy status, monthly population-based rates of COVID-19-associated hospitalizations declined among all adult age groups during the pre-Delta period (Figure 1). Rates subsequently increased during July-August, with the highest rates among adults aged $\geq 65$ years and the lowest among those aged 18-49 years. Monthly ICU admission, IMV, and in-hospital death rates followed the same patterns as COVID-19-associated hospitalization rates by age group,

\footnotetext{
** Fully vaccinated adults with a COVID-19-associated hospitalization were persons who had received the second dose of a 2-dose COVID-19 vaccine series or a single dose of a 1-dose product $\geq 14$ days before receiving a positive SARS-CoV-2 test result associated with their hospitalization. Adults whose positive SARS-CoV-2 test date was $\geq 14$ days after the first dose of a 2-dose series but $<14$ days after receipt of the second dose were considered partially vaccinated. Partially vaccinated adults, and those who received a single dose of a vaccine $<14$ days before the positive SARS-CoV-2 test result were not included in analyses by vaccination status but were included in rates and overall proportions that were not stratified by vaccination status. Adults with no documented receipt of any COVID-19 vaccine dose before the test date were considered unvaccinated. If the SARS-CoV-2 test date was not available, hospital admission date was used. Adults whose vaccination status had not yet been verified using the immunization information system data were considered to have missing vaccination status and were included in total proportions not stratified by vaccination status.

$\dagger \dagger 45$ C.F.R. part 46, 21 C.F.R. part 56; 42 U.S.C. Sect. 241(d); 5 U.S.C. Sect. 552a; 44 U.S.C. Sect. 3501 et seq.
}

with the highest rates in adults aged $\geq 65$ years and the lowest in persons aged $18-49$ years.

During January-August 2021, in a representative sample of 7,615 COVID-19 hospitalizations among nonpregnant adults with detailed clinical data available, $71.8 \%$ (weighted) of patients hospitalized during the Delta period were unvaccinated. Among unvaccinated hospitalized COVID-19 patients, the average monthly proportion who were aged $18-49$ years significantly increased from $26.9 \%$ in the pre-Delta period to $43.6 \%$ during the Delta period ( $\mathrm{p}<0.01$ ) (Table). Among hospitalized COVID-19 patients who were fully vaccinated, the proportion of younger adults did not significantly change between the pre-Delta (10.6\%) and Delta (10.8\%) periods. Among sampled nonpregnant adults hospitalized with COVID-19, no statistically significant differences were observed between the pre-Delta and Delta periods by sex, race/ethnicity, or the proportion of patients who were admitted to an ICU, who received IMV, or who died while hospitalized, overall and stratified by age and vaccination status.

During January-August 2021, the proportion of patients aged $\geq 50$ years hospitalized with COVID-19 who were admitted to an ICU or who died while hospitalized generally trended upward in the Delta period (Figure 2), with the largest increase in persons who died while hospitalized among adults aged $\geq 65$ years, (from $10.2 \%$ in June to $18.1 \%$ in August), although the difference was not statistically significant $(p=0.70)$. Monthly proportions of adults hospitalized with COVID-19 who received IMV also did not change significantly during January-August 2021.

\section{Discussion}

COVID-19-associated hospitalization rates increased after the SARS-CoV-2 Delta variant became predominant. However, the proportion of nonpregnant adults aged $\geq 18$ years hospitalized with COVID-19 who were admitted to an ICU, received IMV, or died during their hospitalization did not significantly change during this period. No significant differences in severity were observed between the pre-Delta and Delta periods among fully vaccinated or unvaccinated hospitalized patients, overall or when stratified by age and vaccination status. However, during the Delta period, adults aged 18-49 years accounted for a larger proportion of hospitalized patients compared with the pre-Delta period. This was driven by the larger number of unvaccinated hospitalized patients in this age group, likely reflecting lower vaccination coverage in younger adults than in older adults.

Similar to this analysis, a previous study examining similar outcomes during March-December 2020 (before Delta variant predominance), found that rates of ICU admission, IMV, and in-hospital death mirrored adult hospitalization rates for that 
FIGURE 1. COVID-19-associated monthly hospitalization rates per 100,000 population among adults aged $\geq 18$ years, ${ }^{*}$ by age group, month,

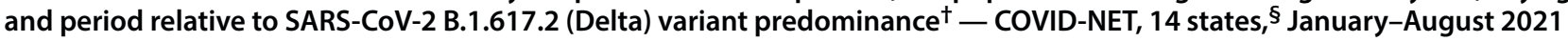

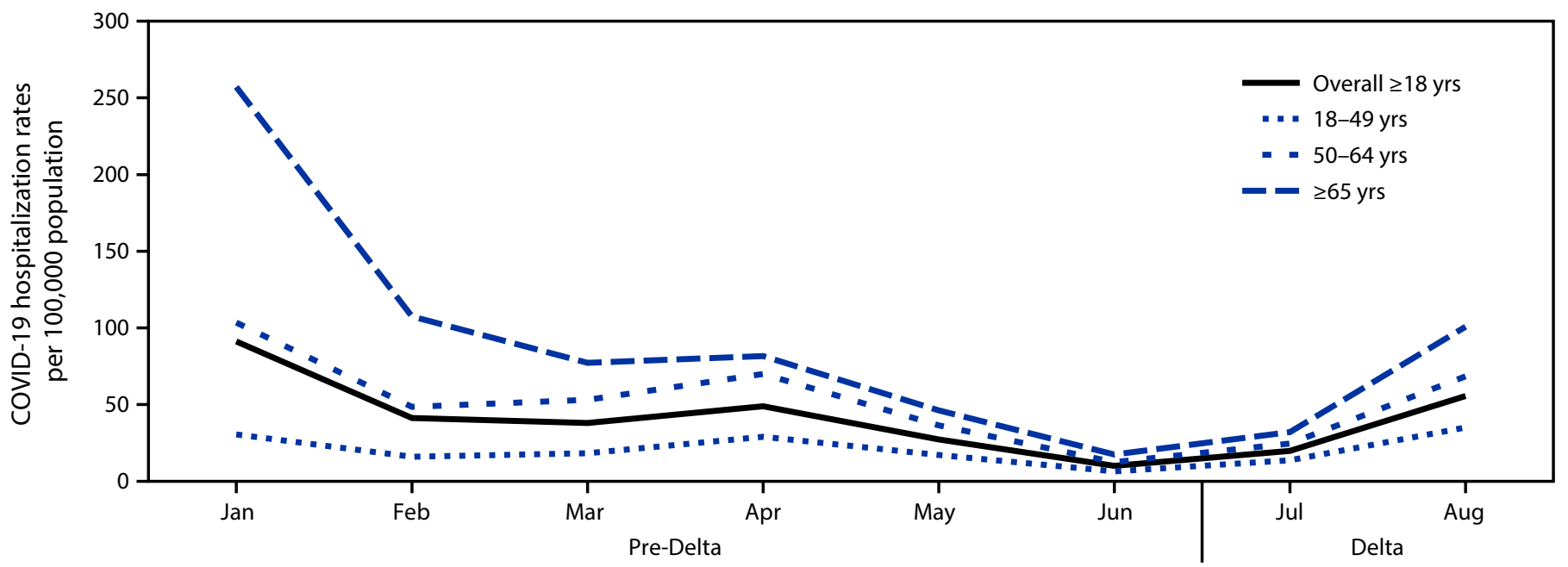

Month

* Proportions are from a weighted sample of hospitalized adults with completed medical chart abstraction and a discharge disposition. Results are subject to change as additional data are reported.

† January-June 2021 is the pre-Delta period; the Delta period (July-August 2021) is when the Delta variant was the predominant circulating variant.

$\S$ Selected counties in California, Colorado, Connecticut, Georgia, lowa, Maryland, Michigan, Minnesota, New Mexico, New York, Ohio, Oregon, Tennessee, and Utah can be found at https://www.cdc.gov/mmwr/volumes/69/wr/mm6915e3.htm.

TABLE. Demographic characteristics and clinical interventions and outcomes among 7,615 nonpregnant adults aged $\geq 18$ years hospitalized with COVID-19, ${ }^{*}$ by vaccination status ${ }^{\dagger}$ and period relative to SARS-CoV-2 B.1.617.2 (Delta) variant predominance ${ }^{\S}-$ COVID-NET, $^{14}$ states, ${ }^{\Uparrow}$ January-August 2021

\begin{tabular}{|c|c|c|c|c|c|c|c|c|c|}
\hline \multirow[b]{3}{*}{ Characteristic } & \multicolumn{9}{|c|}{ Weighted \% of COVID-19 hospitalizations ( $95 \% \mathrm{CI})$} \\
\hline & \multicolumn{3}{|c|}{ Total hospitalizations** } & \multicolumn{3}{|c|}{ Unvaccinated } & \multicolumn{3}{|c|}{ Fully vaccinated } \\
\hline & Pre-Delta period & Delta period & $p$-value ${ }^{\dagger \dagger}$ & Pre-Delta period & Delta period & $p$-value ${ }^{\dagger \dagger}$ & $\dagger$ Pre-Delta period & Delta period & $p$-value ${ }^{\dagger \dagger}$ \\
\hline Total & 5,951 & 1,664 & - & 4,896 & 1,145 & - & 389 & 393 & - \\
\hline \multicolumn{10}{|c|}{ Demographic characteristics $\S \S$} \\
\hline $\begin{array}{l}\text { Age group, yrs } \\
18-49 \\
50-64 \\
\geq 65\end{array}$ & $\begin{array}{l}24.7(23.2-26.3) \\
31.2(29.5-33.0) \\
44.1(42.0-46.2)\end{array}$ & $\begin{array}{l}35.8(32.1-39.5) \\
30.4(27.3-33.7) \\
33.8(30.4-37.4)\end{array}$ & $<0.01$ & $\begin{array}{l}26.9(25.2-28.7) \\
32.4(30.5-34.4) \\
40.6(38.3-43.0)\end{array}$ & $\begin{array}{l}43.6(39.1-48.2) \\
33.6(29.8-37.6) \\
22.8(19.1-26.8)\end{array}$ & $<0.01$ & $\begin{array}{r}10.6(6.8-15.4) \\
17.2(12.9-22.3) \\
72.2(65.8-78.0)\end{array}$ & $\begin{array}{r}10.8(7.1-15.4) \\
18.8(13.6-25.0) \\
70.4(63.6-76.7)\end{array}$ & $>0.99$ \\
\hline $\begin{array}{l}\text { Sex } \\
\text { Male } \\
\text { Female }\end{array}$ & $\begin{array}{l}52.2(50.2-54.3) \\
47.8(45.7-49.8)\end{array}$ & $\begin{array}{l}52.3(48.6-55.9) \\
47.7(44.1-51.4)\end{array}$ & $>0.99$ & $\begin{array}{l}52.4(50.2-54.6) \\
47.6(45.4-49.8)\end{array}$ & $\begin{array}{l}50.5(46.1-55.0) \\
49.5(45.0-53.9)\end{array}$ & $>0.99$ & $\begin{array}{l}51.7(43.8-59.5) \\
48.3(40.5-56.2)\end{array}$ & $\begin{array}{l}56.7(49.3-64.0) \\
43.3(36.0-50.7)\end{array}$ & $>0.99$ \\
\hline $\begin{array}{l}\text { Race/Ethnicity } \\
\text { White } \\
\text { Black } \\
\text { Al/AN } \\
\text { A/PI } \\
\text { Hispanic }\end{array}$ & $\begin{array}{l}\text { ก1 } \\
50.0(47.9-52.0) \\
28.5(26.6-30.5) \\
1.1(0.8-1.4) \\
6.8(5.6-8.2) \\
13.6(12.3-15.0)\end{array}$ & $\begin{array}{r}47.8(44.1-51.6) \\
32.1(28.5-35.9) \\
1.3(0.8-2.0) \\
5.4(3.4-8.1) \\
13.4(10.9-16.2)\end{array}$ & $>0.99$ & $\begin{array}{r}48.5(46.2-50.8) \\
29.6(27.5-31.8) \\
1.0(0.7-1.3) \\
7.1(5.7-8.6) \\
13.8(12.4-15.4)\end{array}$ & $\begin{array}{r}45.4(40.9-49.9) \\
34.0(29.6-38.7) \\
1.2(0.6-2.1) \\
5.0(2.7-8.3) \\
14.4(11.3-18.0)\end{array}$ & $>0.99$ & $\begin{array}{r}65.7(57.7-73.1) \\
16.6(10.4-24.6) \\
1.2(0.4-2.9) \\
5.5(2.5-10.2) \\
11.0(7.3-15.7)\end{array}$ & $\begin{array}{r}57.2(49.2-65.0) \\
23.5(16.8-31.3) \\
1.7(0.6-3.7) \\
6.8(2.8-13.5) \\
10.8(6.4-16.8)\end{array}$ & $>0.99$ \\
\hline $\begin{array}{l}\text { Long-term car } \\
\text { Yes } \\
\text { No }\end{array}$ & $\begin{array}{c}\text { e facility resident* } \\
7.8(6.5-9.1) \\
92.2(90.9-93.5)\end{array}$ & $\begin{array}{r}3.2(2.1-4.5) \\
96.8(95.5-97.9)\end{array}$ & $<0.01$ & $\begin{array}{r}5.9(4.7-7.3) \\
94.1(92.7-95.3)\end{array}$ & $\begin{array}{c}1.6(0.7-3.0)^{\dagger+\dagger} \\
98.4(97.0-99.3)\end{array}$ & $\dagger<0.01$ & $\begin{array}{l}16.7(11.4-23.2) \\
83.3(76.8-88.6)\end{array}$ & $\begin{array}{r}8.3(4.9-12.9) \\
91.7(87.1-95.1)\end{array}$ & 0.59 \\
\hline \multicolumn{9}{|c|}{ Hospitalization interventions and outcomes, by age group, yrs ${ }^{\S \S \S}$} & \\
\hline $\begin{array}{l}\geq 18 \\
18-49 \\
50-64 \\
\geq 65\end{array}$ & $\begin{array}{l}20.1(18.5-21.9) \\
17.1(14.6-19.9) \\
21.4(18.9-24.1) \\
21.0(18.1-24.1)\end{array}$ & $\begin{array}{l}23.4(20.4-26.6) \\
17.1(12.7-22.3) \\
27.8(22.6-33.5) \\
26.2(20.7-32.3)\end{array}$ & $\begin{array}{l}>0.99 \\
>0.99 \\
>0.99 \\
>0.99\end{array}$ & $\begin{array}{l}20.1(18.3-21.9) \\
16.8(14.2-19.6) \\
21.4(18.7-24.4) \\
21.1(17.8-24.8)\end{array}$ & $\begin{array}{l}22.6(19.1-26.3) \\
16.5(11.7-22.2) \\
27.8(22.2-34.0) \\
26.7(18.8-35.9)\end{array}$ & $\begin{array}{l}>0.99 \\
>0.99 \\
>0.99 \\
>0.99\end{array}$ & $\begin{array}{c}19.9(14.2-26.6) \\
\text { - }^{* * * *} \\
18.4(10.2-29.4) \\
19.6(12.7-28.2)\end{array}$ & $\begin{array}{l}24.6(18.2-32.0) \\
32.0(16.5-51.1)^{\dagger+t \dagger} \\
\text { - }^{* * * *} \\
24.2(16.5-33.4)\end{array}$ & $\begin{array}{l}>0.99 \\
>0.99 \\
>0.99 \\
>0.99\end{array}$ \\
\hline
\end{tabular}

See table footnotes on the next page. 
TABLE. (Continued) Demographic characteristics and clinical interventions and outcomes among 7,615 nonpregnant adults aged $\geq 18$ years

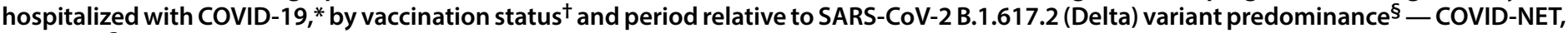
14 states, " January-August 2021

Weighted $\%$ of COVID-19 hospitalizations ( $95 \% \mathrm{Cl}$ )

\begin{tabular}{|c|c|c|c|c|c|c|c|c|c|}
\hline \multirow[b]{2}{*}{ Characteristic } & \multicolumn{3}{|c|}{ Total hospitalizations** } & \multicolumn{3}{|c|}{ Unvaccinated } & \multicolumn{3}{|c|}{ Fully vaccinated } \\
\hline & Pre-Delta period & Delta period & $p$-value ${ }^{t+}$ & Pre-Delta period & Delta period & $p$-value ${ }^{t \dagger}$ & Pre-Delta period & Delta period & $p$-value ${ }^{\dagger \dagger}$ \\
\hline \multicolumn{10}{|c|}{ Invasive mechanical ventilation ${ }^{\S \S \S \S}$} \\
\hline$\geq 18$ & $11.5(10.1-12.9)$ & $11.2(9.1-13.7)$ & $>0.99$ & $11.6(10.1-13.1)$ & $11.3(8.8-14.2)$ & $>0.99$ & $9.4(5.2-15.3)$ & $12.7(7.6-19.5)$ & $>0.99$ \\
\hline $18-49$ & $10.1(8.1-12.4)$ & $7.1(4.2-10.9)$ & $>0.99$ & $9.7(7.7-12.1)$ & $7.2(4.2-11.6)$ & $>0.99$ & - $^{* * * *}$ & $7.4(1.4-21.0)^{\dagger+\dagger}$ & $>0.99$ \\
\hline 50-64 & $11.7(9.8-13.9)$ & $14.5(10.7-19.1)$ & $>0.99$ & $11.7(9.6-14.0)$ & $16.7(12.0-22.2)$ & $>0.99$ & - $^{* * * *}$ & $7.0(2.0-16.8)^{\dagger+\dagger}$ & $>0.99$ \\
\hline$\geq 65$ & $12.1(9.7-14.8)$ & $12.6(8.7-17.6)$ & $>0.99$ & $12.7(10.0-15.9)$ & $11.2(6.6-17.5)$ & $>0.99$ & $7.7(3.3-15.0)$ & $15.0(8.3-24.3)$ & $>0.99$ \\
\hline \multicolumn{10}{|c|}{ In-hospital death } \\
\hline$\geq 18$ & $8.6(7.5-9.9)$ & $9.9(7.9-12.2)$ & $>0.99$ & $8.2(7.0-9.5)$ & $8.7(6.6-11.1)$ & $>0.99$ & $7.2(4.3-11.1)$ & $13.9(8.7-20.7)$ & $>0.99$ \\
\hline $18-49$ & $3.4(2.2-5.0)$ & $2.0(0.7-4.3)^{\dagger+\dagger}$ & $>0.99$ & $3.2(2.0-4.9)$ & $2.1(0.7-4.7)^{\dagger+\dagger}$ & $>0.99$ & $5.0(0.6-16.7)$ & $2.0(0.0-12.8)^{t+\dagger}$ & $>0.99$ \\
\hline $50-64$ & $7.5(5.9-9.3)$ & $9.5(6.4-13.5)$ & $>0.99$ & $7.6(5.8-9.6)$ & $10.5(6.8-15.2)$ & $>0.99$ & $4.2(1.0-11.3)$ & $7.3(1.4-20.7)^{\dagger+\dagger}$ & $>0.99$ \\
\hline$\geq 65$ & $12.3(10.2-14.8)$ & $18.5(13.8-23.9)$ & 0.70 & $12.0(9.6-14.7)$ & $18.6(12.6-25.9)$ & $>0.99$ & $8.2(4.5-13.5)$ & $17.4(10.5-26.3)$ & $>0.99$ \\
\hline
\end{tabular}

Abbreviations: $\mathrm{A} / \mathrm{PI}=$ Asian or Pacific Islander; $\mathrm{Al} / \mathrm{AN}=$ American Indian or Alaska Native; $\mathrm{Cl}=$ confidence interval; ICU = intensive care unit.

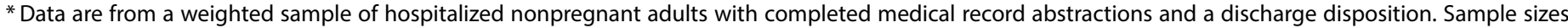
presented are unweighted with weighted percentages.

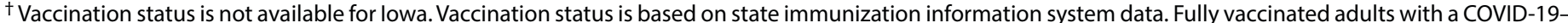

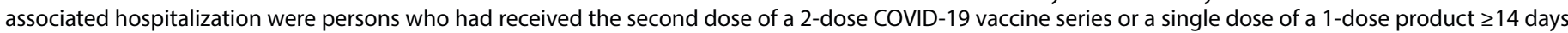

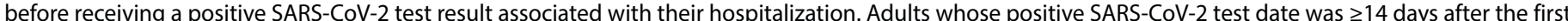

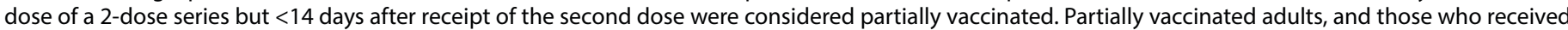

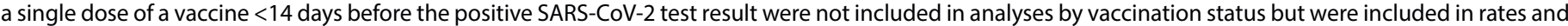

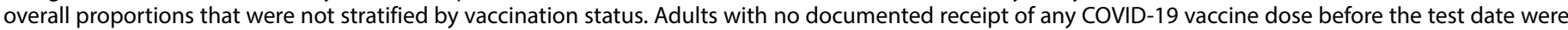

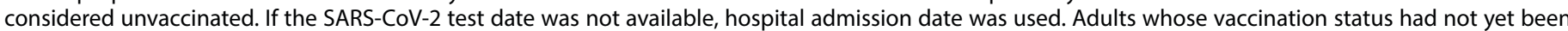

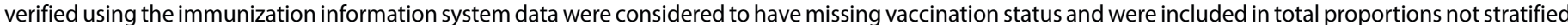

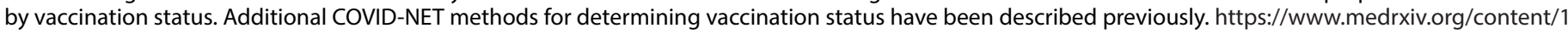
$0.1101 / 2021.08 .27 .21262356 \mathrm{v} 1$

$\S$ January-June 2021 is the pre-Delta period; the Delta period (July-August 2021) is when the Delta variant was the predominant circulating variant.

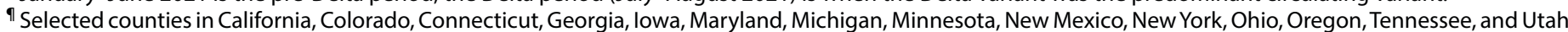
can be found at https://www.cdc.gov/mmwr/volumes/69/wr/mm6915e3.htm.

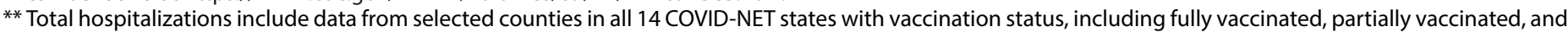
unvaccinated adults. As a result, the number of total hospitalizations exceeds the sum of fully vaccinated and unvaccinated adults.

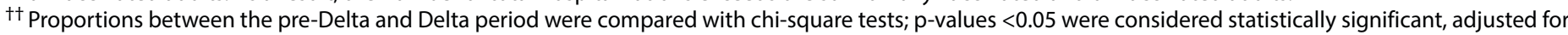
multiple comparisons using the Bonferroni correction method.

$\S \S$ Percentages presented for demographic characteristics are weighted column percentages.

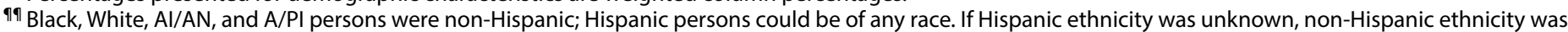

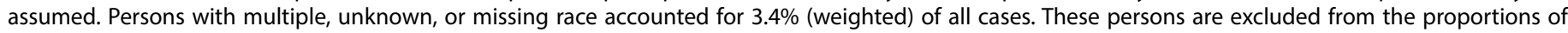
race/ethnicity but are otherwise included elsewhere in the analysis.

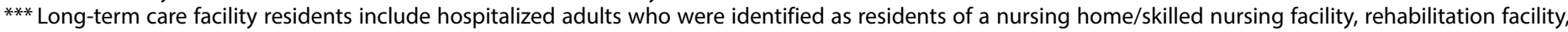

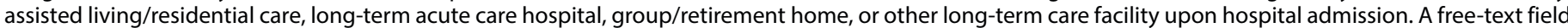
for other types of residences was examined; patients with a long-term care facility-type residence were also categorized as long-term care facility residents.

t†† Relative standard errors $>30 \%$.

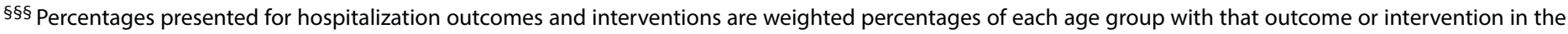
pre-Delta or Delta period.

ๆ ๆ ICU admission status was missing in $0.9 \%$ (weighted) of hospitalizations; these hospitalizations are otherwise included elsewhere in the analysis.

**** Results with relative standard errors $>30 \%$ and $\mathrm{Cl}$ widths $>20$ were suppressed.

t††† Cl widths $>20$.

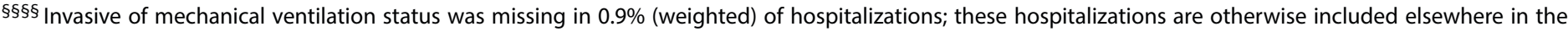
analysis.

ๆางๆ In-hospital death status was missing in 1.2\% (weighted) of hospitalizations; these hospitalizations are otherwise included elsewhere in the analysis.

period (6). These findings are similar to previous analyses of children and adolescents, which showed no significant differences in severe in-hospital outcomes between the pre-Delta and Delta periods $(7,8)$. As rates of infection increased with the Delta variant, other studies have also shown increased risks for associated hospitalization $(9,10)$, and a large Canadian study found an increased risk for ICU admission and death among a cohort of persons infected with the Delta variant (10). However, unlike this analysis, these studies were not limited to persons already hospitalized. Although the increasing trend in hospitalizations resulting in ICU admission or in-hospital death among adults aged $\geq 50$ years was not statistically significant, trends in these outcomes will continue to be examined as outcomes from additional cases in later months of Delta predominance are identified.

Among unvaccinated hospitalized patients, the proportion of adults aged 18-49 years increased during the Delta period while the proportion aged $\geq 65$ years decreased, whereas the 
FIGURE 2. Percentage* of nonpregnant adult patients hospitalized with COVID-19 who were admitted to an intensive care unit and who died while hospitalized, by age group, month, and period relative to SARS-CoV-2 B.1.617.2 (Delta) variant predominance ${ }^{\dagger}-$ COVID-NET, $^{14}$ states, $^{\S}$ January-August 2021

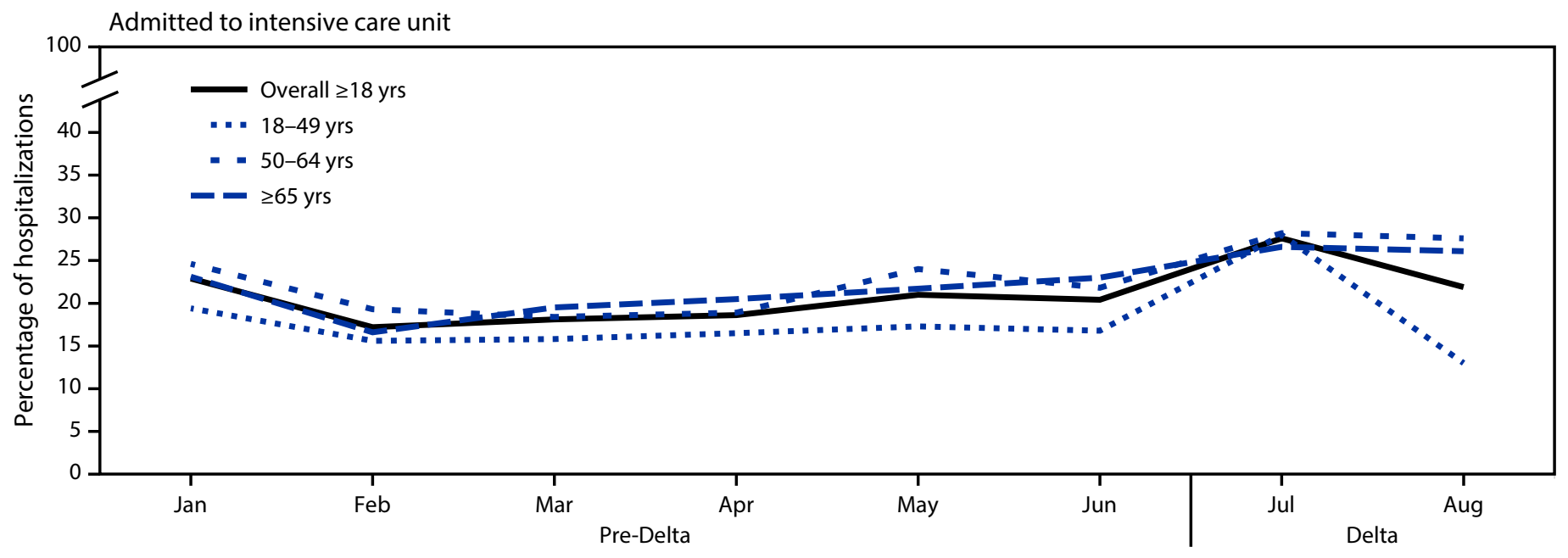

Month

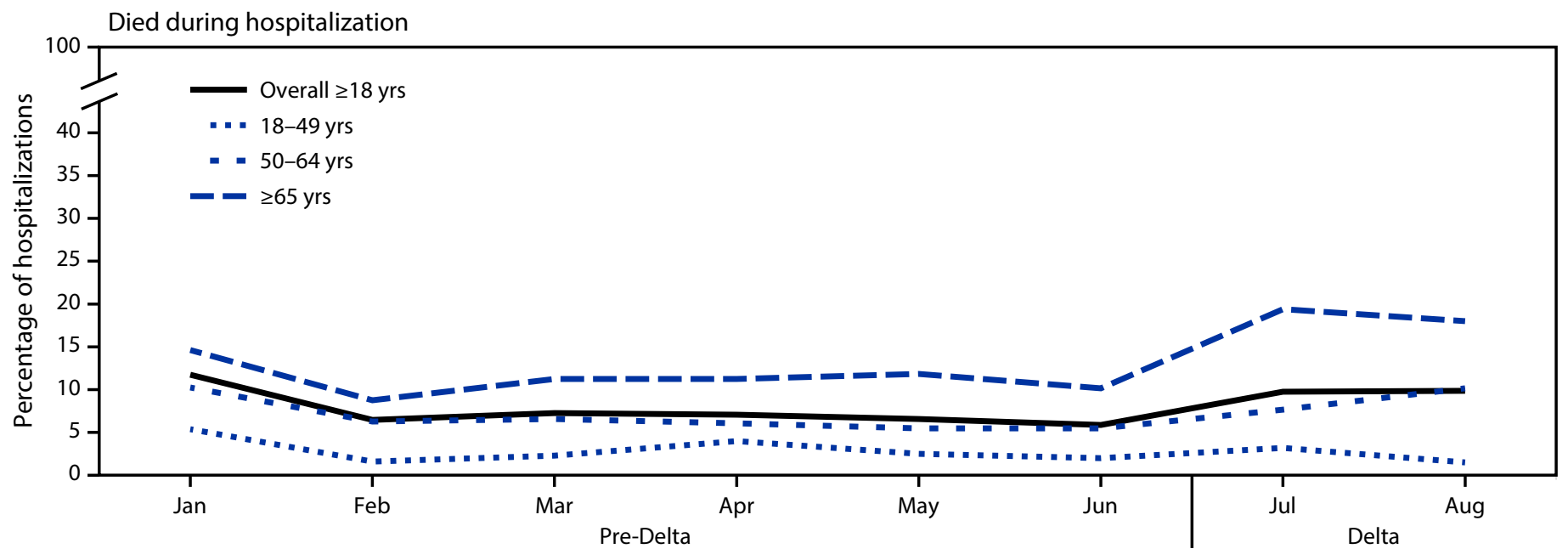

Month

* Proportions are from a weighted sample of hospitalized adults with completed medical chart abstraction and a discharge disposition. Results are subject to change as additional data are reported.

† January-June 2021 is the pre-Delta period; the Delta period (July-August 2021) is when the Delta variant was the predominant circulating variant.

$\S$ Selected counties in California, Colorado, Connecticut, Georgia, lowa, Maryland, Michigan, Minnesota, New Mexico, New York, Ohio, Oregon, Tennessee, and Utah can be found at https://www.cdc.gov/mmwr/volumes/69/wr/mm6915e3.htm.

age distribution among fully vaccinated hospitalized patients remained stable throughout the study period. All age groups included in this study were eligible to receive COVID-19 vaccines; however, as of August 31,2021, the proportion of adults aged $\geq 65$ years who are fully vaccinated $(81.7 \%)$ is far higher than that of adults aged $18-64$ years $(58.6 \%)$. ${ }^{\$ \$}$ Differences in vaccination coverage between age groups possibly contributed to the shift in proportional age distribution of hospitalized patients during the period of Delta predominance.

$\overline{\$ \$}$ https://covid.cdc.gov/covid-data-tracker/\#vaccination-demographic
The findings in this report are subject to at least six limitations. First, COVID-19-associated hospitalizations might be undercounted because testing practices might have resulted in some persons who were admitted but did not receive testing for SARS-CoV-2. Second, the number of hospitalizations among adults aged 18-49 years is relatively small, and ICU admission, receipt of IMV, and in-hospital death are relatively rare outcomes among younger age groups, limiting the ability to examine statistical significance for some outcomes among this age group. Third, the COVID-NET surveillance catchment 
area represents about $10 \%$ of the U.S. population; thus, these findings should not be generalized nationally. Fourth, during periods of increased hospitalization and limited hospital capacity, clinical thresholds for hospitalization and ICU admission might shift and could potentially obscure trends in increased severity. Fifth, the analysis did not account for the propensity of persons to be vaccinated, and therefore could not determine the effectiveness of vaccination in reducing severe outcomes. Finally, data presented are preliminary and might change as additional cases are identified and reported, including cases from July and August that do not yet have a discharge disposition. 99

Rates of COVID-19-associated hospitalizations in adults increased during July-August 2021 as the Delta variant became predominant in the United States. Although this variant is more transmissible, this study did not find significantly higher proportions of hospitalizations with ICU admission, receipt of IMV, or in-hospital death in nonpregnant hospitalized adults. The proportion of unvaccinated adults aged $18-49$ years hospitalized with COVID-19 has increased as the Delta variant has become more predominant. COVID-19 vaccination is critical for all eligible adults, including those aged $<50$ years who have relatively low vaccination rates compared with older adults.

\footnotetext{
99 For hospitalizations with admission dates during July-August 2021, 1.3\% of cases are missing a discharge diagnosis.
}

\section{Acknowledgments}

Roxanne Archer, Kareena Hundal, Sherry Quach, Maria Rosales, Lena Tayo, Tiffany Tsukuda, California Emerging Infections Program; Ann Basting, Tessa Carter, Maria Correa, Daewi Kim, Carol Lyons, Amber Maslar, Julie Plano, Connecticut Emerging Infections Program, Yale School of Public Health; Katelyn Ward, Jana Manning, Asmith Joseph, Allison Roebling, Chandler Surell, Stephanie Lehman, Taylor Eisenstein, Gracie Chambers, Suzanne Segler, Grayson Kallas, Marina Bruck, Rayna Ceaser, Georgia Emerging Infections Program, Georgia Department of Health, Veterans Affairs Medical Center, Foundation for Atlanta Veterans Education and Research; Jim Collins, Shannon Johnson, Sue Kim, Libby Reeg, Alexander Kohrman, Lauren Leegwater, Sierra PeguiesKhan, Chloe Brown, Michigan Department of Health and Human Services; Austin Bell, Kayla Bilski, Emma Contestabile, Claire Henrichsen, Amanda Gordon, Cynthia Kenyon, Melissa McMahon, Katherine Schleiss, Samantha Siebman, Emily Holodnick, Lisa Nguyen, Kristen Ehresmann, Jennifer Gilbertson, Jill Reaney, Alison Babb, Grace Hernandez, Kieu My Phi, Minnesota Department of Health; Daniel Sosin, Sunshine Martinez, Jasmyn Sanchez, Susan L. Ropp, Cory Cline, New Mexico Department of Health; Sarah Lathrop, Kathy M. Angeles, Emily B. Hancock, Yadira SalazarSanchez, Sarah A. Khanlian, Nancy Eisenberg, Melissa Christian,

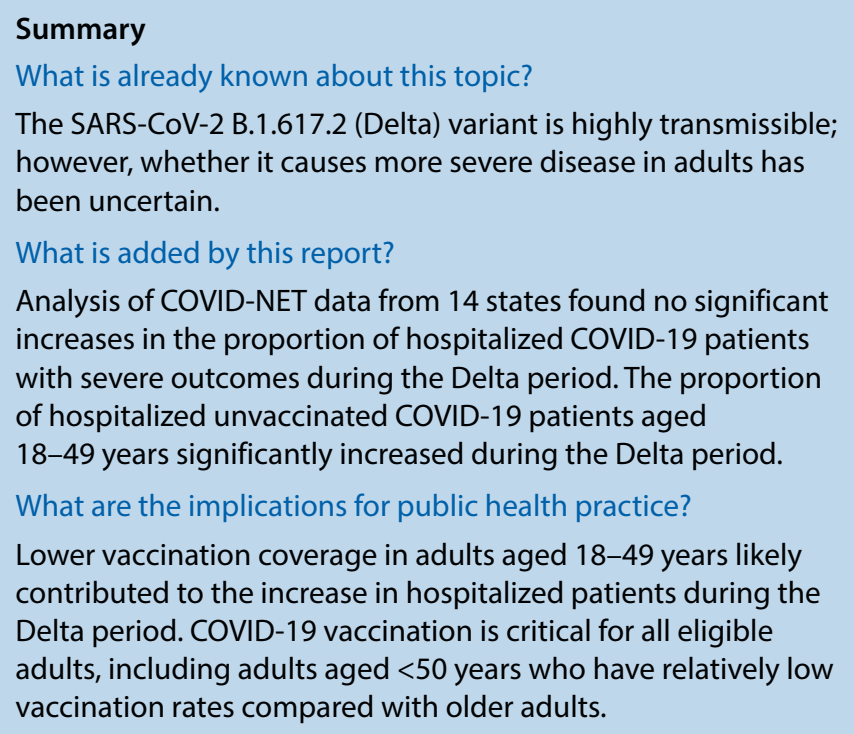

Dominic Rudin, Sarah Shrum Davis, Chelsea McMullen, New Mexico Emerging Infections Program; Yassir Talha, Murtada Khalifa, CDC Foundation, New Mexico Department of Health; Alison Muse, Suzanne McGuire, New York State Department of Health; Sophrena Bushey, Christina Felsen, Christine Long, University of Rochester School of Medicine and Dentistry; Ama Owusu-Dommey, Breanna McArdle, Public Health Division, Oregon Health Authority; Kylie Seeley, Oregon Health \& Science University School of Medicine; Karen Lei, Katie Dyer, Terri McMinn, Danielle Ndi, Anise Elie, Kathy Billings, Manideepthi Pemmaraju, John Ujwok, Vanderbilt University Medical Center; Amanda Carter, Andrea George, Andrew Haraghey, Ashley Swain, Caitlin Shaw, Ian Buchta, Laine McCullough, Mary Hill, Ryan Chatelain, Tyler Riedesel, Salt Lake County Health Department.

\section{COVID-NET Surveillance Team}

Jeremy Roland, California Emerging Infections Program, Oakland, California; David Blythe, Maryland Department of Health; Alicia Brooks, Maryland Department of Health; Kathryn Como-Sabetti, Minnesota Department of Health; Richard Danila, Minnesota Department of Health; Melissa Judson, New Mexico Department of Health; Wickliffe Omondi, New Mexico Emerging Infections Program; Kerianne Engesser, New York State Department of Health; Adam Rowe, New York State Department of Health; Maria Gaitán, University of Rochester School of Medicine and Dentistry, Rochester, New York; Virginia Cafferky, University of Rochester School of Medicine and Dentistry, Rochester, New York; Julie Freshwater, Ohio Department of Health; Ann Salvator, Ohio Department of Health; Sam Hawkins, Public Health Division, Oregon Health Authority; Emily Youngers, Public Health Division, Oregon Health Authority; Tiffanie Markus, Vanderbilt University Medical Center, Nashville, Tennessee; Melanie Crossland, Salt Lake County Health Department, Salt Lake City, Utah; Keegan McCaffrey, Utah Department of Health. 
Corresponding author: Christopher A. Taylor, cataylor1@cdc.gov.

${ }^{1}$ CDC COVID-19 Response Team; ${ }^{2}$ General Dynamics Information Technology, Atlanta, Georgia; ${ }^{3}$ California Emerging Infections Program, Oakland, California; ${ }^{4}$ Career Epidemiology Field Officer Program, CDC; ${ }^{5}$ Colorado Department of Public Health and Environment; ${ }^{6}$ Connecticut Emerging Infections Program, Yale School of Public Health, New Haven, Connecticut; ${ }^{7}$ Emory University School of Medicine, Atlanta, Georgia; ${ }^{8}$ Georgia Emerging Infections Program, Georgia Department of Health; ${ }^{9}$ Atlanta Veterans Affairs Medical Center, Atlanta, Georgia; ${ }^{10}$ Iowa Department of Public Health; ${ }^{11}$ Maryland Department of Health; ${ }^{12}$ Michigan Department of Health and Human Services; ${ }^{13}$ Minnesota Department of Health; ${ }^{14} \mathrm{New}$ Mexico Emerging Infections Program, University of New Mexico, Albuquerque, New Mexico; ${ }^{15}$ New Mexico Department of Health; ${ }^{16}$ New York State Department of Health; ${ }^{17}$ University of Rochester School of Medicine and Dentistry, Rochester, New York; ${ }^{18}$ Rochester Emerging Infections Program, University of Rochester Medical Center, Rochester, New York; ${ }^{19}$ Ohio Department of Health; ${ }^{20}$ Public Health Division, Oregon Health Authority; ${ }^{21}$ Vanderbilt University Medical Center, Nashville, Tennessee; ${ }^{22}$ Salt Lake County Health Department.

All authors have completed and submitted the International Committee of Medical Journal Editors form for disclosure of potential conflicts of interest. Evan J. Anderson reports grants from Pfizer, Merck, PaxVax, Micron, Sanofi-Pasteur, Janssen, MedImmune, and GlaxoSmithKline; personal fees from Pfizer, Medscape, Kentucky Bioprocessing, Inc., Sanofi-Pasteur, and Janssen, outside the submitted work; and institutional funding from the National Institutes of Health to conduct clinical trials of Moderna and Janssen COVID-19 vaccines. Laurie M. Billing and Eli Shiltz report grants from the Council of State and Territorial Epidemiologists during the conduct of the study. Ruth Lynfield reports editorial fees from the American Academy of Pediatrics Red Book (Committee on Infectious Diseases), which were donated to the Minnesota Department of Health. William Schaffner reports personal fees from VBI Vaccines, outside the submitted work. No other potential conflicts of interest were disclosed.

\section{References}

1. Dougherty K, Mannell M, Naqvi O, Matson D, Stone J. SARS-CoV-2 B.1.617.2 (Delta) variant COVID-19 outbreak associated with a gymnastics facility - Oklahoma, April-May 2021. MMWR Morb Mortal Wkly Rep 2021;70:1004-7. PMID:34264910 https://doi.org/10.15585/ mmwr.mm7028e2

2. Garg S, Patel K, Pham H, et al. Clinical trends among U.S. adults hospitalized with COVID-19, March to December 2020: a crosssectional study. Ann Intern Med 2021;174:1409-19. PMID:34370517 https://doi.org/10.7326/M21-1991

3. Delahoy MJ, Whitaker M, O'Halloran A, et al.; COVID-NET Surveillance Team. Characteristics and maternal and birth outcomes of hospitalized pregnant women with laboratory-confirmed COVID-19COVID-NET, 13 states, March 1-August 22, 2020. MMWR Morb Mortal Wkly Rep 2020;69:1347-54. PMID:32970655 https://doi. org/10.15585/mmwr.mm6938e1

4. Thompson MG, Burgess JL, Naleway AL, et al. Prevention and attenuation of Covid-19 with the BNT162b2 and mRNA-1273 vaccines. N Engl J Med 2021;385:320-9. PMID:34192428 https:// doi.org/10.1056/NEJMoa2107058

5. Moline HL, Whitaker M, Deng L, et al. Effectiveness of COVID-19 vaccines in preventing hospitalization among adults aged $\geq 65$ yearsCOVID-NET, 13 states, February-April 2021. MMWR Morb Mortal Wkly Rep 2021;70:1088-93. PMID:34383730 https://doi. org/10.15585/mmwr.mm7032e3

6. Havers FP, Pham H, Taylor CA, et al. COVID-19-associated hospitalizations among vaccinated and unvaccinated adults $\geq 18$ yearsCOVID-NET, 13 states, January 1-July 24, 2021. medRxiv [Preprint posted online August 29, 2021]. https://www.medrxiv.org/content/10. $1101 / 2021.08 .27 .21262356 \mathrm{v} 1$

7. Delahoy MJ, Ujamaa D, Whitaker M, et al.; COVID-NET Surveillance Team. Hospitalizations associated with COVID-19 among children and adolescents-COVID-NET, 14 states, March 1, 2020-August 14, 2021. MMWR Morb Mortal Wkly Rep 2021;70:1255-60. PMID:34499627 https://doi.org/10.15585/mmwr.mm7036e2

8. Siegel DA, Reses HE, Cool AJ, et al.; MAPW1. Trends in COVID-19 cases, emergency department visits, and hospital admissions among children and adolescents aged 0-17 years-United States, August 2020August 2021. MMWR Morb Mortal Wkly Rep 2021;70:1249-54. PMID:34499628 https://doi.org/10.15585/mmwr.mm7036e1

9. Twohig KA, Nyberg T, Zaidi A, et al.; COVID-19 Genomics UK (COGUK) consortium. Hospital admission and emergency care attendance risk for SARS-CoV-2 delta (B.1.617.2) compared with alpha (B.1.1.7) variants of concern: a cohort study. Lancet Infect Dis 2021;S14733099(21)00475-8 Published online October 13, 2021. PMID:34461056

10. Fisman DN, Tuite AR. Evaluation of the relative virulence of novel SARS-CoV-2 variants: a retrospective cohort study in Ontario, Canada. CMAJ 2021;cmaj.211248. PMID:34610919 https://doi.org/10.1503/ cmaj. 211248 